\section{REFERENCES}

1 Suissa S, Ernst P, Vandemheen KL, Aaron SD. Methodological issues in therapeutic trials of COPD. Eur Respir J 2008; 31: 927-933.

2 Sin DD, Wu L, Anderson JA, et al. Inhaled corticosteroids and mortality in chronic obstructive pulmonary disease. Thorax 2005; 60: 992-997.

3 Calverley PM, Anderson JA, Celli B, et al. Salmeterol and fluticasone propionate and survival in chronic obstructive pulmonary disease. $N$ Engl J Med 2007; 356: 775-789.
4 Aaron SD, Vandemheen KL, Fergusson D, et al. Tiotropium in combination with placebo, salmeterol, or fluticasonesalmeterol for treatment of chronic obstructive pulmonary disease: a randomized trial. Ann Intern Med 2007; 146: 545-555.

5 Wedzicha JA, Calverley PM, Seemungal TA, et al. The prevention of chronic obstructive pulmonary disease exacerbations by salmeterol/fluticasone propionate or tiotropium bromide. Am J Respir Crit Care Med 2008; 177: 19-26.

DOI: $10.1183 / 09031936.00030508$

\title{
Prior tuberculin skin testing does not boost QuantiFERON-TB results in paediatric contacts
}

\section{To the Editors:}

We read with interest the paper by LEYTEN et al. [1], which appeared in the June 2007 issue of the European Respiratory Journal (ERJ). LEYTEN et al. [1] showed that prior tuberculin skin tests (TST) do not induce false-positive QuantiFERON ${ }_{\mathbb{R}}-\mathrm{TB}$ Gold in-tube (QFT-GIT) assay results when evaluated in the days immediately following TST administration. In the same issue of the ERJ, NASEER et al. [2] reported the results of a study of 10 subjects without risk for tuberculosis infection, who were tested with QFT-GIT and the T-SPOT.TB assay (Oxford Immunotec, Abingdon, UK) before, $48 \mathrm{~h}$ and 6 weeks after TST administration. NASEER et al. [2] confirmed the results of LEYTEN et al. [1] in the first days following skin testing; however, after 6 weeks, three out of nine individuals turned from negative to positive with QFT-GIT, and none of the subjects turned from negative to positive with T-SPOT.TB. On this basis, NASEER et al. [2] state that there is evidence of boosting-specific interferon (IFN)- $\gamma$ responses 6 weeks after TST. According to these results, a high incidence of falsepositive results with QFT-GIT should be expected when the assay is performed weeks after the skin test. This issue is potentially very relevant in clinical practice, as current guidelines indicate that contacts recently exposed should be tested for latent infection at the time of diagnosis of the index case and, if negative, after 8-12 weeks [3]. We therefore retrospectively reviewed our series of paediatric contacts to identify an effect similar to the one reported by NASEER et al. [2].

A total of 70 children received both TST and QuantiFERON ${ }_{\mathbb{B}-}$ TB Gold (QFT-G); 51 (72.9\%) were QFT-G negative, nine (12.8\%) were QFT-G positive and 10 (14.3\%) were QFT-G indeterminate (low positive control). After 8-11 weeks, only one of the 51 initially QFT-G-negative contacts became QFT-G positive, and the mean values of IFN $-\gamma$ units produced after stimulation with early secretory antigenic target (ESAT)- 6 and culture filtrate protein (CFP)-10 did not differ before and after TST (ESAT-6 difference: $0.005 \mathrm{IU} \cdot \mathrm{mL}^{-1}, \quad \mathrm{p}=0.745 ; \mathrm{CFP}-10$ difference: $\left.0.030 \mathrm{IU} \cdot \mathrm{mL}^{-1}, \mathrm{p}=0.453\right)$. Of the 10 children with indeterminate QFT-G results, six tested indeterminate again, three became negative and one became positive. A total of 81 children were evaluated with TST and QFT-GIT. At the first visit, $63(77.8 \%)$ contacts were QFT-GIT negative, eight $(9.9 \%)$ were QFT-GIT positive and $10(12.3 \%)$ had an undetermined QFT-GIT. Of those initially negative children, only one became QFT-GIT positive after TST. The mean IFN- $\gamma$ antigen-specific production did not change 11 weeks after skin testing (ESAT-6/ CFP-10/TB7.7 difference $-0.030 \mathrm{IU} \cdot \mathrm{mL}^{-1}, \mathrm{p}=0.281$ ). Of the 10 subjects who were initially indeterminate with QFT-GIT, six remained indeterminate and four tested negative (table 1). Interestingly, as already reported in other studies [4, 5], we detected imperfect agreement between TST and QFT; in particular, the presence of TST-positive, QFT-negative children is similar to data recently published in an adult population [6]. Our findings show that among children at risk of acquiring latent tuberculosis infection after contact with an infectious index case, there is minimal or no effect on QFT several weeks after initial skin testing. Only two household contacts became QFT positive and both were heavily exposed to smear-positive cases; these QFT conversions could represent true recent infections, rather than sensitisation to purified protein derivative. Conversely, such a low rate of QFT conversions might be considered unexpected, and could be explained by the fact that at the time of index case diagnosis, most children already had prolonged contact and therefore may have undergone an antigen-specific T-cell response. At initial evaluation, $\sim 10 \%$ of contacts were QFT positive.

Our findings are consistent with those reported by LEYTEN et al. [1] but are in disagreement with those of NASEER et al. [2]; differences in size, demographic and clinical characteristics of study groups may account for this discrepancy. Our experience with IFN- $\gamma$ release assays for the diagnosis of latent tuberculosis supports the concept that prior administration of TST does not induce false-positive results of the blood tests. The lack of boosting effect of repeated TST on the enzymelinked immunospot-based blood assay has already been reported in 44 bacille Calmette-Guerin-unvaccinated TSTnegative adult and paediatric contacts repeatedly tested with the skin and blood assay over a 2 -yr period [7]. In that study, three conversions were observed with TST and none were observed with the blood assay. 


\begin{tabular}{|c|c|c|c|c|c|c|}
\hline \multirow[t]{3}{*}{ TABLE 1} & $\begin{array}{l}3 \text { Gold (QF } \\
\text { le of re-tes }\end{array}$ & QuantiF & $N_{\mathbb{R}}-\mathrm{TB}$ Gold & pe (QFT & d tuber & kin tests (TST) \\
\hline & \multicolumn{3}{|c|}{ QFT-G } & \multicolumn{3}{|c|}{ QFT-GIT } \\
\hline & Negative & Positive & Indeterminate & Negative & Positive & Indeterminate \\
\hline $\mathbf{M} / \mathbf{F}$ & 29/22 & $2 / 7$ & $4 / 6$ & $22 / 41$ & $4 / 4$ & $6 / 4$ \\
\hline$B C G$ vaccinated & 15 & 3 & 1 & 14 & 1 & 0 \\
\hline Results at initial screening & 51 & 9 & 10 & 63 & 8 & 10 \\
\hline Results after 8-11 weeks & 53 & 11 & 6 & 66 & 9 & 6 \\
\hline TST $\mathrm{mm}$ at initial screening & $1.7 \pm 4.3$ & $8.0 \pm 4.3$ & $0.3 \pm 0.9$ & $1.1 \pm 3.9$ & $8.5 \pm 6.4$ & 0 \\
\hline TST mm after 8-11 weeks & $1.7 \pm 4.3$ & ND & $0.6 \pm 1.3$ & $1.4 \pm 3.9$ & ND & 0 \\
\hline
\end{tabular}

Overall, our results support the combined use of the skin and the blood assays, as indicated by recent guidelines [8]. However, it remains to be established whether this strategy is the most effective for the use of these new promising diagnostic tests.

\section{Richeldi*, B.M. Bergamini" ${ }^{\#}$ and F. Vaienti ${ }^{\#}$}

Depts of *Respiratory Diseases, "Paediatrics, University of Modena and Reggio Emilia, Modena, Italy.

\section{STATEMENT OF INTEREST}

None declared.

\section{REFERENCES}

1 Leyten EMS, Prins C, Bossink AWJ, et al. Effect of tuberculin skin testing on a Mycobacterium tuberculosis-specific interferon- $\gamma$ assay. Eur Respir J 2007; 29: 1212-1216.

2 Naseer A, Naqvi S, Kampmann B. Evidence for boosting Mycobacterium tuberculosis-specific IFN- $\gamma$ responses at 6 weeks following tuberculin skin testing. Eur Respir J 2007; 29: $1282-1283$.
3 Targeted tuberculin testing and treatment of latent tuberculosis infection. Am J Respir Crit Care Med 2000; 161: S221-S247.

4 Ferrara G, Losi M, D’Amico R, et al. Use in routine clinical practice of two commercial blood tests for diagnosis of infection with Mycobacterium tuberculosis: a prospective study. Lancet 2006; 367: 1328-1334.

5 Kang YA, Lee HW, Yoon HI, et al. Discrepancy between the tuberculin skin test and the whole-blood interferon $\gamma$ assay for the diagnosis of latent tuberculosis infection in an intermediate tuberculosis-burden country. JAMA 2005; 293: 2756-2761.

6 Mazurek GH, Zajdowicz MJ, Hankinson AL, et al. Detection of Mycobacterium tuberculosis infection in United States Navy recruits using the tuberculin skin test or whole-blood interferon- $\gamma$ release assays. Clin Infect Dis 2007; 45: 826-836.

7 Richeldi L, Ewer K, Losi M, Roversi P, Fabbri LM, Lalvani A. Repeated tuberculin testing does not induce false positive ELISPOT results. Thorax 2006; 61: 180.

8 National Collaborating Centre for Chronic Condistions. Tuberculosis: clinical diagnosis and management of tuberculosis, and measures for its prevention and control. London, Royal College of Physicians, 2006.

DOI: 10.1183/09031936.00014508 